\title{
A simulation analysis of train rescheduling strategies on Chinese passenger dedicated lines
}

\author{
Z. $\mathrm{He}^{1}$, L. Meng ${ }^{2}, \mathrm{H} . \mathrm{Li}^{2} \& \mathrm{~L} . \mathrm{Nie}^{2}$ \\ ${ }^{1}$ State Key Lab. of Rail Traffic Control and Safety, \\ Beijing Jiaotong University, China \\ ${ }^{2}$ School of Traffic and Transportation, Beijing Jiaotong University, China
}

\begin{abstract}
Train rescheduling plays a vital role in railway operation. Many factors should be considered in the train rescheduling process and train class, delayed time, remaining distance and current position are the most common ones. There are usually different measures aiming at corresponding factors and combinations of the measures with different priorities compose various train rescheduling strategies. Furthermore, different strategies lead diverse results and they are applicable to various scenarios respectively. This paper researched the effects of different train rescheduling strategies in the background of Wuguang passenger dedicated line by means of simulation experiments. It analyzed class-based strategies first and achieved the suitable strategy and its related parameters. Then it analyzed the influence of different combinations of high-speed strategies and middle-speed strategies under different perturbation scenarios.
\end{abstract}

Keywords: train rescheduling strategy; passenger dedicated line; simulation.

\section{Introduction}

Train rescheduling is the key work in railway operation. Its basic idea is to determine a new order of the trains when some perturbations make current schedule disordered and infeasible. Many factors should be considered together and corresponding measures will be taken in this process. The measures and their various combinations compose different train rescheduling strategies which will probably lead diverse results and are applicable to various scenarios respectively.

Because of high density and high speed of trains, train rescheduling work in Chinese Passenger Dedicated Lines (PDL) is more difficult than that in 
traditional lines. In addition, the mix-speed operation mode that high-speed trains and middle-speed trains run on the line simultaneously will be applied in many of these lines and will last for many years, which will increase the difficulty of train rescheduling much further. Due to too many factors involved in the process of train rescheduling, effectiveness of each strategy can hardly be calculated by pure mathematical ways. Therefore, computer simulation becomes the common way for analysis of train rescheduling strategies. Nie [1] and Zhang [2] analyzed impact factors in train rescheduling by simulation and gave some good ideas. Jin [3] also analyzed the dispatchers' preferences which would be helpful to the research of the train rescheduling strategies.

This paper will analyze the influence of different strategies on train rescheduling in Chinese PDL by means of computer simulation. The analysis of simulation data under different strategies will provide foundations for reasonable and effective train rescheduling strategies in Chinese PDL.

\section{Analysis of train rescheduling strategies}

When perturbations occur, some conflicts will generally take place which will make current schedule infeasible. To resolve the conflicts and get new optimized schedules composed the main work of train dispatchers. The common approach is to resolve each conflict from the earliest one and rearrange order of trains pair by pair, as shown in Figure 1. Some researchers had developed the algorithms in accordance to this idea [4-6].

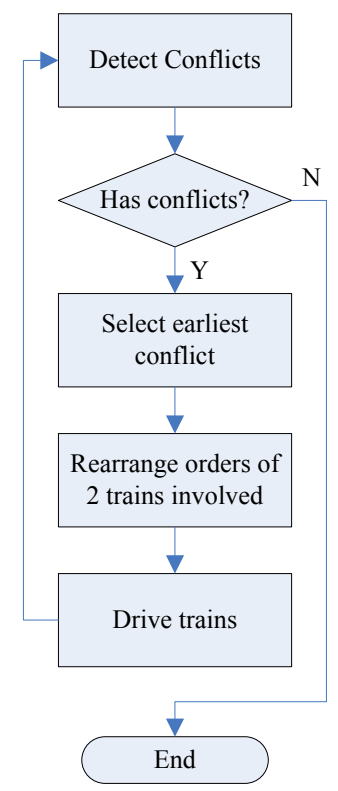

Figure 1: Process of train rescheduling. 
In this process, new order will be assigned to involved trains according to their characteristics and, in which train class, delayed time and remaining journey are the most common ones. Some strategies are hereby created and applied to determine which train will depart firstly.

Train class is usually a primary factor in train rescheduling, which makes the class-based rescheduling strategies- severe strategy and flexible strategy- become the basic strategies in train rescheduling. Severe strategy (Strategy P0) means that higher-class trains will undoubtedly depart first. Flexible strategy (Strategy P1) means that lower-class trains can depart first in some cases.

Then various strategies are designed aiming at delayed time and remaining journey respectively in order to study the influence of respective strategies on the trains with different classes in PDL. Under the precondition of class-based rescheduling (strategy P0 or P1), the strategies will be designed as following:

If the trains involved in the current conflict have different class, strategy P0 or P1 will be applied to determine which train has higher priority, otherwise, one of the following 5 strategies may be used:

1) Strategy 1: The trains will depart in accordance with their current order and all the other factors will be ignored.

2) If both of the trains involved in the current conflict are delayed, they will depart in accordance with their current order. However, if one of the trains is delayed and another is punctual, then the strategy will be:

Strategy 2: The punctual train will always depart first.

Strategy 3: The delayed train can depart first only if delayed train run ahead of punctual train currently and the time difference between the earliest possible departure time of the delayed train and punctual train is less than I/2 (where I is the standard headway interval time). In any other cases, the punctual train will depart first.

3) If the difference of remaining journey between the two trains is less than $M$ minutes where $M$ is the running time of about 2-3 sections, they will depart according to current order. Else, the following strategies will be applied:

Strategy 4: The train with longer remaining journey has the higher priority and will depart first.

Strategy 5: The train with shorter remaining journey has the higher priority and will depart first.

\section{Simulation analysis of class-based strategies}

The framework of the simulation is shown as figure 2. Simulation control module controls occurrence of various perturbations, simulation iterations, kinds of data analyzed and some other parameters. The strategies control module will determine which strategy should be applied in current scenario. The train advancing module is in charge of movement of trains and train primary delay will come into being in this process. The rescheduling module will detect and resolve each conflict in turn in the process of train advancing according to different strategies loaded by strategy control module. Finally, the data statistics and analysis module will collect and analyze simulation results when simulations 


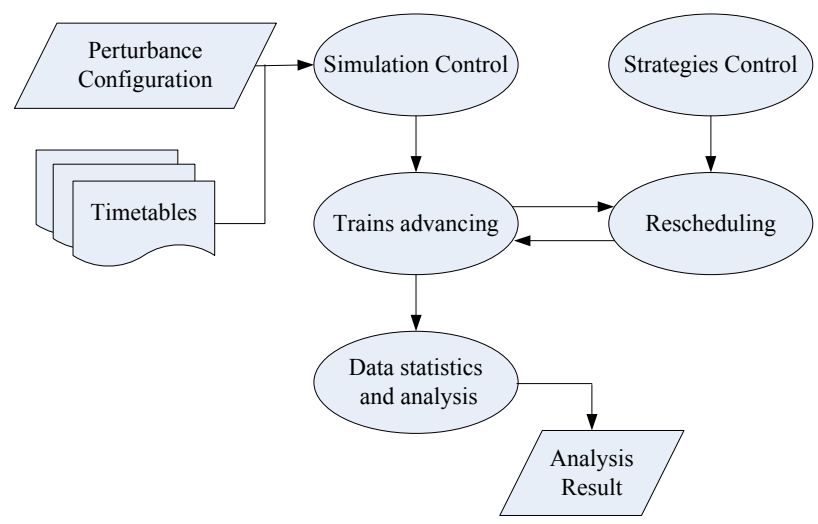

Figure 2: Framework of the simulation.

aiming at each strategy have been accomplished. The detailed approach of train advancing and rescheduling can be found in reference [6] and [7].

In this study, Wuguang PDL (from Wuhan to Guangzhou) was taken as example. A timetable including 115 pairs of high-speed trains $\left(1^{\text {st }}\right.$ class, $300-350$ $\mathrm{km} / \mathrm{h}$ ) and 30 pairs of middle-speed trains ( $2^{\text {nd }}$ class, $200-250 \mathrm{~km} / \mathrm{h}$ ) was made for the simulation. High-speed trains are distributed into the timetable perioduniformly which means the quantity of the trains per hour is different in different period (morning/ evening rush hours, normal time, etc.) but same within a period. The middle-speed trains are assigned in the timetable also uniformly with the density of 1-2 trains per hour except in rush hours (7:00-9:00 and 17:0018:00). Considering the relative abundant capacity of this line, the minimum interval between two trains in the timetable is set to $I+1$ in order to ensure enough buffer for train rescheduling. In addition, the allowance of the running time is set to $6 \%-7 \%$.

As mentioned above, there are 2 types of class-based strategies. The difference between them is that whether the lower-class trains are permitted to influence higher-class trains. In fact, the two strategies can be concluded into one strategy as: when a conflict occurs between a higher-class train and a lower-class train, the higher-class train can be moved only if it ran behind of the lower-class train currently and the moved time is no more than $N$ minutes. If $N=0$, the strategy is severe strategy, or it's the flexible one. Obviously, $N$ is the key parameter of class-based strategies.

First, $N$ is set to 0 and the initial delay time of middle-speed trains which are created by simulation control module is fitted to normal distribution with expected value of 5, 10, 15, 20 (minutes) respectively. Meanwhile, there is no delay with high-speed trains. In the process of train rescheduling, class-based strategy is applied, and then the strategies 1-5 are applied to the middle-speed trains respectively. The results are shown in table 1. 
Table 1: Results of middle-speed train rescheduling with different initial delay time.

\begin{tabular}{|c|c|c|c|c|c|c|}
\hline $\begin{array}{l}\text { AIDT/ } \\
\text { QIDT* }\end{array}$ & Strategy & 1 & 2 & 3 & 4 & 5 \\
\hline \multirow{3}{*}{$5: 00 / 1.9$} & QFDT & 0.9 & 0.8 & 1 & 0.9 & 0.9 \\
\hline & AFDT & $27: 55$ & $40: 24$ & $34: 57$ & $29: 53$ & $32: 09$ \\
\hline & TFDT & $24: 59$ & $32: 57$ & $33: 35$ & $26: 21$ & $28: 56$ \\
\hline \multirow{3}{*}{$10: 25 / 2$} & QFDT & 1.8 & 1.5 & 1.6 & 1.8 & 1.8 \\
\hline & AFDT & $39: 58$ & $52: 10$ & $48: 13$ & $40: 22$ & $41: 45$ \\
\hline & TFDT & $1: 10: 53$ & 1:17:01 & $1: 15: 46$ & $1: 12: 33$ & $1: 13: 34$ \\
\hline \multirow{3}{*}{$15: 08 / 2$} & QFDT & 2 & 1.7 & 1.8 & 2.1 & 2 \\
\hline & AFDT & $43: 56$ & $58: 54$ & $54: 24$ & $43: 48$ & $45: 30$ \\
\hline & TFDT & $1: 29: 28$ & $1: 37: 29$ & $1: 36: 30$ & $1: 30: 45$ & $1: 31: 32$ \\
\hline \multirow{3}{*}{$19: 32 / 2$} & QFDT & 2.4 & 1.9 & 2 & 2.5 & 2.4 \\
\hline & AFDT & $43: 12$ & $58: 33$ & $54: 35$ & $42: 45$ & $45: 29$ \\
\hline & TFDT & $1: 44: 55$ & $1: 51: 15$ & $1: 50: 28$ & $1: 45: 52$ & $1: 47: 14$ \\
\hline
\end{tabular}

*AIDT: Average Initial Delay Time;

QFDT: Quantity of Finally Delayed Trains QIDT: Quantity of Initial delayed trains; AFDT: Average Final Delay Time TFDT: Total Final Delay Time

As we can see from table 1, when AIDT is greater than 10 minutes, the result turns worse remarkably. The reason is that the middle-speed trains will generally enter the time slots of high-speed trains and can hardly recover their delay time. So ten minutes is an important critical value and will be used in the following research.

Since then, the study will continue to simulate the cases in which $5 \%$ of middle-speed trains will delay ten minutes averagely and $N=0,1,3,5,7$ minutes. In the process, strategy 1 will be applied in both middle-speed and high-speed train rescheduling. The result is shown in table 2 .

Table 2: $\quad$ Influence of $N$ on rescheduling results.

\begin{tabular}{rrrrrrr}
\hline & $\mathrm{N}$ & \multicolumn{1}{c}{0} & \multicolumn{1}{c}{1} & \multicolumn{1}{c}{3} & \multicolumn{1}{c}{5} & \multicolumn{1}{c}{7} \\
\hline $\begin{array}{c}\text { Middle- } \\
\text { speed } \\
\text { trains }\end{array}$ & QFDT & 1.8 & 1.1 & 0.6 & 0.5 & 0.5 \\
& AFDT & $39: 58$ & $26: 10$ & $19: 58$ & $21: 17$ & $18: 46$ \\
& TFDT & $1: 10: 53$ & $28: 40$ & $12: 36$ & $11: 39$ & $09: 49$ \\
\hline $\begin{array}{l}\text { High- } \\
\text { speed } \\
\text { trains }\end{array}$ & QFDT & 0 & 0.3 & 0.4 & 0.5 & 1.1 \\
& AFDT & 0 & $02: 13$ & $02: 23$ & $02: 38$ & $03: 36$ \\
\hline
\end{tabular}


The data above show that all the indices of middle-speed trains, especially QFDT and TFDT, have been improved to a quite large extent with the increase of $N$. When the value of $N$ changes from 0 to 1 and 1 to 3 minutes, TFDT of middle-speed trains increases obviously. However, when the value of $N$ continues to grow up, the increase of TFDT slows down. When the value of $N$ changes from 5 to 7 minutes, the result of high-speed trains turns worse evidently. It indicates that there's a reasonable range of value for $N$. Considering the change of indices of high-speed and middle-speed trains synthetically, 3 to 5 minutes is a suitable value to the strategy in this study. In addition, the real interval between trains in the timetable of current study is about 4.5 minutes $(I+1)$.

The analysis above shows that the value of $N$ should be a little less than the real interval between high-speed trains. If $N$ is more than real interval between high-speed trains in the timetable, a high-speed train is possibly moved behind the other one, which will result that the train can hardly recover from delay. The quality of railway operation will turn worse severely along with it. Since then, the value of 3 minutes is selected as the value of $N$ in the following research.

\section{Simulation analysis on different combinations of high-speed strategies and middle-speed strategies under different perturbation scenarios}

Twelve scenarios of perturbations (train delay) are made after analyzing the current operation of Chinese railway and shown as table 3 .

Table 3: $\quad$ Scenarios of perturbations.

\begin{tabular}{ccccc}
\hline \multirow{2}{*}{ No } & \multicolumn{2}{c}{ High-speed trains } & \multicolumn{2}{c}{ Middle-speed trains } \\
\cline { 2 - 5 } & Probability $\%$ & $\begin{array}{c}\text { Delay Time } \\
(\text { min })\end{array}$ & Probability/\% & $\begin{array}{c}\text { Delay Time } \\
(\text { min })\end{array}$ \\
\hline 1 & 3 & 5 & 0 & 0 \\
2 & 0 & 0 & 5 & 5 \\
3 & 0 & 0 & 5 & 10 \\
4 & 0 & 0 & 5 & 15 \\
5 & 3 & 5 & 5 & 5 \\
6 & 3 & 5 & 5 & 15 \\
7 & 3 & 5 & 10 & 5 \\
8 & 3 & 5 & 10 & 10 \\
9 & 5 & 5 & 10 & 15 \\
10 & 5 & 5 & 20 & 15 \\
11 & 5 & 10 & 20 & 15 \\
12 & 10 & 15 & 20 & 15 \\
\hline
\end{tabular}



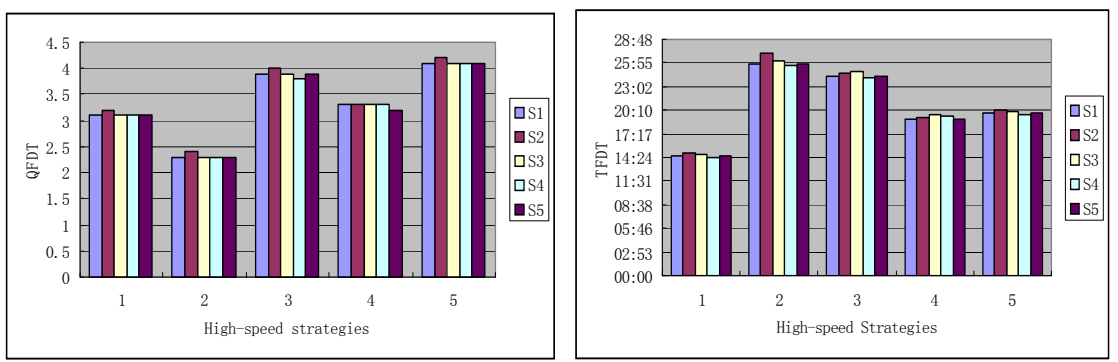

(a) High-speed trains
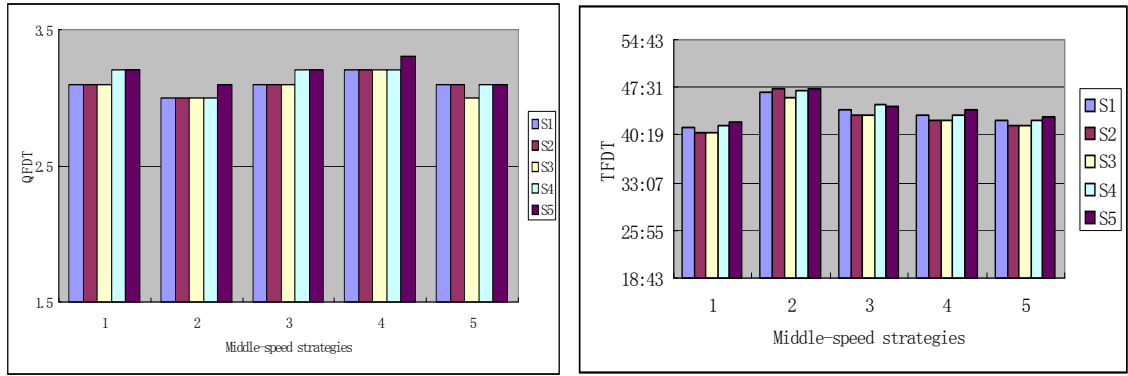

(b) Middle-speed trains

Figure 3: $\quad$ Change of QFDT and TFDT.

After a number of simulation experiments, we got many useful data and found some interesting facts.

As to each scenario, different combinations of high-speed and middle-speed train rescheduling strategies will be applied with the precondition of strategy P1 ( $N=3$ minutes). It means that there are 25 strategy combinations applied to each scenario since there are 5 types of strategies for high-speed and middle-speed trains respectively.

1) The interference between a middle-speed strategy and a high-speed strategy is very little in any scenario. We take the result of scenario 8 as example. In this scenario, the QIDT and AIDT of high-speed are 3.6 and 5:20 and them of middle-speed trains are 3.4 and 10:00. The change of QFDT and TFDT of high-speed trains are shown in figure 3(a) in which S1-S5 represent current middle-speed train rescheduling strategies 1-5 and the number of $\mathrm{x}$-axis is current high-speed strategy.

As we can see from the figure 3(a), as to the same high-speed train rescheduling strategy, when the middle strategy is changed, the value of QFDT and TFDT almost keep invariable. (The maximum change of QFDT and TFDT are 0.2 trains and $1: 33$ )

Now we continue to research the influence of high-speed strategies on middle-speed strategies. The example is also scenario 8. The change of QFDT and TFDT of middle-speed trains are shown in figure 3(b). 
The change of QFDT and TFDT of middle-speed trains is also very little when high-speed strategy is changed, only 0.2 trains and 1:26 respectively.

Moreover, the characteristics about interference in other scenarios are very similar to scenario 8 . Therefore, the interference between middle-speed strategies and high-speed strategies is little in any scenario. So we can generally pay no attention to another type of strategies when we focus on high-speed or middlespeed train rescheduling strategies.

2) In the view of TFDT of high-speed trains, strategy 1 has the best result according to the data in table 4 , which is also right in other scenarios. It means that strategy 1 can make better use of time allowance arranged in timetable than other strategies. Therefore, strategy 1 should be recommended in high-speed train rescheduling.

Table 4: $\quad$ Result of high-speed trains rescheduling under several scenarios.

\begin{tabular}{|c|c|c|c|c|c|c|c|}
\hline Scenarios & $\begin{array}{l}\text { QIDT/ } \\
\text { AIDT }\end{array}$ & $\begin{array}{l}\text { High-speed } \\
\text { Strategies }\end{array}$ & 1 & 2 & 3 & 4 & 5 \\
\hline \multirow{3}{*}{1} & \multirow{3}{*}{$\begin{array}{l}3.6 / \\
5: 20\end{array}$} & QFDT & 1.8 & 1.1 & 2.2 & 1.9 & 2.2 \\
\hline & & AFDT & $05: 01$ & $17: 25$ & $07: 41$ & $06: 32$ & $05: 23$ \\
\hline & & TFDT & 08:52 & $19: 38$ & $16: 51$ & $12: 39$ & $12: 01$ \\
\hline \multirow{3}{*}{5} & \multirow{3}{*}{$\begin{array}{c}3.6 / \\
5: 20\end{array}$} & QFDT & 2.1 & 1.6 & 2.8 & 2.2 & 2.8 \\
\hline & & AFDT & $05: 14$ & $13: 30$ & $06: 57$ & $06: 50$ & $05: 12$ \\
\hline & & TFDT & 11:00 & $21: 42$ & $19: 14$ & $15: 20$ & $14: 41$ \\
\hline \multirow{3}{*}{8} & \multirow{3}{*}{$\begin{array}{l}3.6 / \\
5: 20\end{array}$} & QFDT & 3.1 & 2.3 & 3.9 & 3.3 & 4.1 \\
\hline & & AFDT & $04: 42$ & $11: 05$ & $06: 13$ & $05: 49$ & 04:49 \\
\hline & & TFDT & $14: 32$ & $25: 46$ & $24: 14$ & $19: 00$ & $19: 46$ \\
\hline \multirow{3}{*}{12} & \multirow{3}{*}{$\begin{array}{c}12.3 / \\
15: 04\end{array}$} & QFDT & 18.9 & 12.6 & 17.8 & 19.6 & 20.1 \\
\hline & & AFDT & 09:20 & $20: 51$ & $11: 46$ & 10:07 & $09: 25$ \\
\hline & & TFDT & $2: 56: 41$ & $4: 22: 22$ & $3: 29: 17$ & $3: 18: 56$ & $3: 09: 19$ \\
\hline
\end{tabular}

3) The DFDT of high-speed trains of strategy 2 is the least one. So strategy 2 can be applied if the objective of train rescheduling is to minimize the quantity of delayed trains. However, when we turn our eyes to TFDT, the result of strategy 2 is the worst one.

The following work is to research the influence of different strategies on middle-speed train rescheduling. Table 5 shows the result of middle-speed train rescheduling in scenario 5, 8 and 12 .

1) Comparing each AIDT with its corresponding AFDT, it will be found that when middle-speed trains are delayed, their delay time will increase obviously regardless of the strategies applied.

2) The difference of the results is very little when various strategies are applied to the same scenario. 
Table 5: $\quad$ Result of middle-speed trains rescheduling under several scenarios.

\begin{tabular}{|c|c|c|c|c|c|c|c|}
\hline Scenarios & $\begin{array}{l}\text { QIDT/ } \\
\text { AIDT }\end{array}$ & $\begin{array}{l}\text { Middle- } \\
\text { speed } \\
\text { Strategies }\end{array}$ & 1 & 2 & 3 & 4 & 5 \\
\hline \multirow{3}{*}{5} & \multirow{3}{*}{$1.7 / 4: 57$} & QFDT & 1.2 & 1.2 & 1.2 & 1.2 & 1.3 \\
\hline & & AFDT & $08: 45$ & $07: 55$ & $08: 16$ & 08:48 & 08:55 \\
\hline & & TFDT & $10: 31$ & $09: 50$ & $10: 02$ & $10: 19$ & $11: 16$ \\
\hline \multirow{3}{*}{8} & \multirow{3}{*}{$\begin{array}{c}3.4 / 10: 0 \\
0\end{array}$} & QFDT & 3.1 & 3 & 3.1 & 3.2 & 3.1 \\
\hline & & AFDT & $13: 11$ & $15: 39$ & $14: 08$ & $13: 34$ & $13: 55$ \\
\hline & & TFDT & $41: 26$ & $46: 50$ & 44:09 & $43: 20$ & $42: 28$ \\
\hline \multirow{3}{*}{12} & \multirow{3}{*}{$\begin{array}{c}6.7 / 15: 3 \\
2\end{array}$} & QFDT & 9.9 & 9.5 & 9.8 & 9.9 & 9.8 \\
\hline & & AFDT & $16: 38$ & $18: 45$ & $17: 21$ & $16: 46$ & $16: 57$ \\
\hline & & TFDT & $2: 44: 22$ & $2: 57: 25$ & $2: 50: 30$ & $2: 46: 44$ & $2: 45: 50$ \\
\hline
\end{tabular}

The two facts above are also right in the other scenarios. They mean that the rescheduling of middle-speed trains is still limited severely although strategy P1 is applied. If we want to achieve better results, the value of $N$ must increase further. However, it will lead to worsen high-speed train rescheduling results remarkably.

\section{Conclusions}

The paper researched the influence of different train rescheduling strategies designed by train classes, delayed time and the remaining journey on train operation in passenger dedicated line under the operation mode with both highspeed and middle-speed trains by means of simulation. The simulation results showed that:

1) If the middle-speed trains are permitted to move the high-speed trains, the TFDT will decrease evidently, in other words, their anti-disturbance ability will increase remarkably. The compelling moving time of high-speed trains should be a little less than the real interval between high-speed trains in timetable in order to improve the results of middle-speed trains rescheduling and keep the influence on high-speed trains quit little at the same time.

2) The interference between middle-speed strategy and high-speed strategy in the same process of train rescheduling is little in any scenario. So we can generally pay no attention to another type of strategies when we focus on highspeed or middle-speed train rescheduling strategies.

3) In the view of finally delay time of high-speed trains, strategy 1 has the best rescheduling result and should be recommended in high-speed train rescheduling. The DFDT of high-speed trains of strategy 2 becomes the least among all strategies, but the overall final delay time of strategy 2 is the worst. 
4) The rescheduling of middle-speed trains is still limited severely by highspeed trains although strategy P1 is applied. So the difference of the result is very little when various strategies are applied to same scenario.

The rescheduling strategy involves lots of factors and the relations among them are very complicated. Besides the factors researched in this paper, the strategy has also close relations with many other factors, especially the parameters of timetable. Hence, the influence of the mode of timetable designing and the values of all kinds of parameters of timetable on rescheduling strategy will be the important work for the following research.

\section{Acknowledgement}

This study was sponsored by State Key Lab of Rail Traffic Control \& Safety, Beijing Jiaotong University (No.RCS2009ZT008). The authors deeply appreciate the support.

\section{References}

[1] Lei Nie, etc. (2001), Study on strategy of train operation adjustment on high speed railway. Journal of the China railway society, 23(4), pp. 1-6

[2] Xingchen Zhang (1998), A simulation analysis of middle speed train delay influence under the operation mode with high and middle speed train in Jinhu high speed railway, Journal of the China railway society, 20(5), pp. 1-6

[3] Fucai Jin (2004), Study on Theory and Methods of Multi-Objective Optimization of Train Operation Adjustment, Doctor Dissertation, Beijing Jiaotong Unitversity.

[4] J. Medanic, M.J. Dorfman (2004), Scheduling trains on a railway network using a discrete event model of railway traffic, Transportation Research Part B, 38, pp. 81-98

[5] Ismail Sahin (1999), Railway traffic control and train scheduling based on inter-train conflict management, Transportation Research Part B, 33, pp. 511-534

[6] Zhenhuan He (2005), Study on adjustment method of operation diagram with computer, railway computer application, 14(10), pp. 4-6

[7] Zhenhuan He, etc (2009), Research on Greedy Train Rescheduling Algorithm, Proceedings of the 9th ICCTP, ASCE, Harbin: Harbin Institute of Technology. 